ARTIGO ORIGINAL ORIGINAL ARTICLE

\title{
Uma abordagem econômica de processos judiciais de medicamentos impetrados contra um município do sul do Brasil
}

\author{
An economic analysis of the lawsuits filed \\ against municipality in southern Brazil.
}

Alexandra Ferro de Mello', Letícia Santana da Silva Soares², Camila Alves Areda ${ }^{3}$, Carine Raquel Blatt ${ }^{4}$, Dayani Galato

DOI: 10.21115/JBES.v8.n1.p39-46

\section{Palavras-chave:}

assistência farmacêutica, acesso aos serviços de saúde, direito à saúde, uso de medicamentos, economia da saúde

\section{Keywords:}

pharmaceutical services, access to health services, right to health, medicines utilization, health economics

\section{RESUMO}

Objetivo: Apresentar uma análise econômica dos processos judiciais impetrados contra um município de médio porte. Métodos: Estudo descritivo retrospectivo com base na análise de documentos dos processos judiciais impetrados no ano de 2010 contra o município. Resultados: A maioria dos processos era representada por advogados particulares e continha prescrição de médicos provenientes do sistema privado. A antecipação de tutela foi concedida em $88,8 \%$ dos casos. $O$ gasto por medicamento por paciente pela via judicial foi em torno de 200 vezes maior do que o gasto com medicamentos do Componente Básico da Assistência Farmacêutica. 50,0\% do custo total de medicamentos adquiridos pela via judicial foram gastos com agentes antineoplásicos e imunomoduladores, mas estes foram disponibilizados a apenas $8,0 \%$ dos pacientes litigantes. Medicamentos presentes no componente especializado da Assistência Farmacêutica e medicamentos inovadores representaram, respectivamente, $36,8 \%$ e $16,0 \%$ do custo total. Conclusões: A solicitação de medicamentos via judicial tem um custo médio por paciente muito mais elevado do que os medicamentos do Componente Básico da Assistência Farmacêutica e parte significativa deste custo está associado à solicitação de medicamentos novos, bem como a antineoplásicos e imunossupressores.

\begin{abstract}
Objective: To present an economic analysis of the lawsuits filed against medium-sized municipality. Methods: A retrospective descriptive study based on documents analysis from lawsuits filed in 2010 against a municipality. Results: Most of legal actions were represented by private attorneys and contained prescriptions provided by private physicians. Advance relief was granted to $88.8 \%$ of these actions. The drug/patient cost through the courts was around 200 times higher than expenses with basic component drugs of pharmaceutical care. 50.0\% of the total cost with drugs acquired through the courts were represented by antineoplastic agents and immunomodulators drugs, which were provided to only $8.0 \%$ of the litigant patients. Drugs present in the specialized pharmaceutical assistance component and innovative drugs represented, respectively, 36.8\% and $16.0 \%$ of the total cost. Conclusions: Drugs requested by legal proceedings have an average cost per patient much higher if compared to the basic component of pharmaceutical care. Significant portion of this cost is related to the new drug application, as well as antineoplastic agents and immunomodulators drugs.
\end{abstract}

Recebido em: 03/11/2015. Aprovado para publicação em: 07/12/2015

1. Mestranda, Universidade do Sul de Santa Catarina, Centro de Ciências da Saúde, Tubarão, Santa Catarina, Brasil.

2. Mestranda, Universidade de Brasília, Programa de Pós-Graduação em Ciências e Tecnologias em Saúde, Brasília, DF, Brasil.

3. Professora, Universidade de Brasília, Distrito Federal, Brasil.

4. Professora, Universidade Federal de Saúde de Porto Alegre; Porto Alegre, Rio Grande do Sul, Brasil.

5. Professora, Universidade de Brasília, Programa de Pós-Graduação em Ciências e Tecnologias em Saúde, Brasília, DF, Brasil.

Potenciais conflitos de interesse: Nada a declarar.

Fontes de financiamento do estudo: Não houve.

Originado do trabalho de dissertação de Alexandra Ferro de Melo, Universidade do Sul de Santa Catarina, 2013.

Autor para Correspondência: Dayani Galato - Programa de Pós-Graduação em Ciências e Tecnologias em Saúde, Universidade de Brasília - Centro Metropolitano, conjunto A, lote 01, Prédio Unidade de Ensino e Docência (UED) - Ceilândia Sul - Brasília - DF. CEP: 72220-900. E-mail: dayani.galato@gmail.com Telefone/FAX: (061)3107-8416. 


\section{Introdução}

A judicialização da saúde, que inclui os medicamentos, remete à reflexão sobre o impasse de se privilegiar o individual em detrimento do coletivo, permitindo desta forma o questionamento do quanto e de como são gastos os recursos financeiros na aquisição de medicamentos "sub judice" no Sistema Único de Saúde (SUS).

Ressalta-se que, além do fato dos medicamentos constituírem insumo importante para realização do cuidado à saúde, eles requerem adequada gestão dos recursos alocados para a sua aquisição, bem como seleção apropriada daqueles que serão incorporados no SUS (Paim et al, 2011; Pepe et al, 2010; Macedo, Lopes \& Barberato-Filho, 2011). Uma vez que os recursos da saúde são finitos, estes são critérios fundamentais para garantir a sustentabilidade do financiamento público (Paim et al, 2011; Vieira, 2008; Vieira 2009).

Vários estudos têm sido desenvolvidos no Brasil apresentando o perfil dos processos e dos pacientes que impetram ações contra as diferentes esferas de gestão do SUS (Pereira et al, 2010; Ronsein, 2010; Messeder, Osorio-de-Castro \& Luiza, 2005; Romero, 2008; Leite et al, 2009; Biehl et al, 2012). Estes trabalhos, de uma maneira geral, apresentam duas realidades. A primeira relacionada a problemas de acesso quando da solicitação de medicamentos contidos em listas padronizadas por entes públicos e; a segunda de solicitação de medicamentos não padronizados. Neste último caso, são inúmeras as situações encontradas e entre elas pode-se citar a solicitação de medicamentos não classificados como essenciais sob o ponto de vista da sociedade ou dos entes governamentais, aqueles que se caracterizam como inovações tecnológicas geralmente registrados há pouco tempo no país, os gerados por condutas discordantes dos protocolos clínicos definidos no SUS e, demandas motivadas por tratamentos não cobertos no setor complementar por planos de saúde (Pandolfo, Delduque \& Amaral, 2012; Reveiz et al, 2013).

Mesmo que alguns autores discutam a dificuldade de financiamento no SUS e sua relação com a judicialização (Vieira, 2008; Vieira, 2009; Prado, 2013), são poucos os estudos que realizam uma análise econômica deste cenário. Sendo assim, é importante determinar a proporção da judicialização no orçamento da saúde nas diferentes esferas de governo, orientando a tomada de decisões contribuindo para uma distribuição mais racional dos recursos disponíveis, visando possibilitar uma orientação política e de gestão para solução do problema do financiamento da Assistência Farmacêutica (Pepe et al, 2010). O efeito destas demandas sobre o SUS já é perceptível e são necessárias, portanto, ações para reduzir este impacto (Prado, 2013).

Neste contexto, o presente trabalho tem como objetivo apresentar uma abordagem econômica dos processos judiciais impetrados contra o município de Tubarão-SC.

\section{Métodos}

Foi realizado um estudo descritivo retrospectivo com base na análise de documentos dos processos judiciais movidos contra o Município de Tubarão-SC e vigentes no período de $1^{\circ}$ de janeiro a 31 de dezembro de 2010. Foram excluídos os processos que, mesmo possuindo medicamentos, não demandaram estes insumos no período investigado, aqueles que continham apenas outros produtos para saúde e aqueles em duplicidade.

O município de Tubarão tem uma população de cerca de 98.000 habitantes (Censo de 2010) sendo classificado como médio porte, está localizado na região sul de Santa Catarina e é sede da Associação dos Municípios da Região de Laguna (AMUREL), formada por 17 municípios. A distância de Florianópolis, capital do Estado de Santa Catarina é de aproximadamente 140 quilômetros ao sul. Tubarão é a segunda maior cidade em população do sul do Estado, sendo importante pólo de saúde, com dois hospitais, uma clínica médica e a rede de atendimento público municipal (com 31 equipes da estratégia de saúde da família), além da Clínica Integrada na Universidade do Sul de Santa Catarina, que possui atendimento vinculado com as Secretarias Municipais da Região da AMUREL.

Os elementos processuais foram obtidos da análise dos processos judiciais disponíveis na Secretaria Municipal de Saúde. Entre os elementos processuais foram investigados: réu da ação; representação jurídica; vínculo do prescritor; tempo de vigência do processo; quantidade de processos com tutela antecipada; presença da descrição do problema de saúde e; da Classificação Internacional de Doenças e Problemas Relacionados à Saúde (CID-10). É importante destacar que quando o problema de saúde não estava classificado segundo a CID-10, este foi descrito como situações não classificadas.

Os medicamentos solicitados foram classificados, adotando-se o primeiro nível referente ao grupo anatômico principal, através da classificação Anatomical Therapeutic Chemical (ATC). A presença nas listas de medicamentos padronizadas como a Relação Nacional de Medicamentos Essenciais (RENAME) e a Relação Municipal dos Medicamentos (REMUME), os medicamentos do Componente Especializado da Assistência Farmacêutica (CEAF) e os medicamentos do Programa Farmácia Popular foram verificadas comparando-se o medicamento solicitado (princípio ativo, forma farmacêutica e concentração) com aqueles descritos nas listas em vigência no ano de 2010. Quando se observou diferentes apresentações de um mesmo medicamento, este foi considerado parcialmente presente nas listas, desde que apresentasse a mesma finalidade terapêutica.

A avaliação da prescrição dos medicamentos quanto à nomenclatura genérica foi realizada observando-se a Denominação Comum Brasileira ou a Denominação Comum Internacional. 
O registro na Agência Nacional de Vigilância Sanitária Anvisa e sua respectiva data de inscrição foram verificados através do sítio da Agência Reguladora na internet (www. portal.anvisa.gov.br). Quanto à data de registro, foram considerados novos medicamentos aqueles com registro inferior a cinco anos (registrados após 2004). A pesquisa foi realizada adotando-se o nome comercial do medicamento presente no processo. No caso de solicitação por nome genérico, a consulta foi realizada pelo medicamento genérico mais antigo registrado com aquela substância, ou do produto comercial mais antigo, quando da ausência de genérico.

Para a análise econômica, foram solicitados os dados relacionados ao gasto para a aquisição de medicamentos no Município de Tubarão, através da Secretaria de Gestão Municipal, para o exercício de 2010, bem como os valores praticados na última compra dos medicamentos "sub judice" naquele período.

Foi realizado o cálculo dos valores necessários para a aquisição da quantidade deferida de medicamentos "sub judice". Também foi calculado o percentual de gastos em cada uma das proporções identificadas nas listas padronizadas e nas classes terapêuticas de acordo com o grupo anatômico principal da ATC, e dos medicamentos considerados novos. Neste caso, não foram considerados para fins de cálculo os medicamentos parcialmente presentes.

O banco de dados foi criado no programa Excel e após a aquisição destas informações e sua respectiva tabulação iniciou-se a análise descritiva das informações.

O projeto foi aprovado pelo Comitê de Ética em Pesquisa da Universidade do Sul de Santa Catarina sob código 11.068.4.01.III.

\section{Resultados}

No período estudado, segundo dados fornecidos pela Secretaria de Saúde em 2010, havia 327 pastas com processos judiciais vigentes. Após a aplicação dos critérios de seleção (Figura 1), resultaram 209 pastas a serem analisadas. Destas pastas, observaram-se 14 pacientes com dois processos individuais, bem como três processos coletivos (com dois pacientes). Nesse contexto, foram analisados 223 processos diferentes, que representavam 212 pacientes. O que representa 2 processos de solicitação de medicamentos via judicial a cada 1000 habitantes.

As características dos processos incluídos nesta pesquisa são apresentadas na Tabela 1. As ações foram deferidas, em sua grande maioria, com antecipação de tutela $(88,8 \%)$, sendo que nas demais não foi possível identificar se o deferimento foi com tutela antecipada, visto que as pastas encontravam-se com visto judicial incompleto.

Dos 221 problemas de saúde identificados, verificou-se que estes estão distribuídos em 15 classificações do CID-10 e

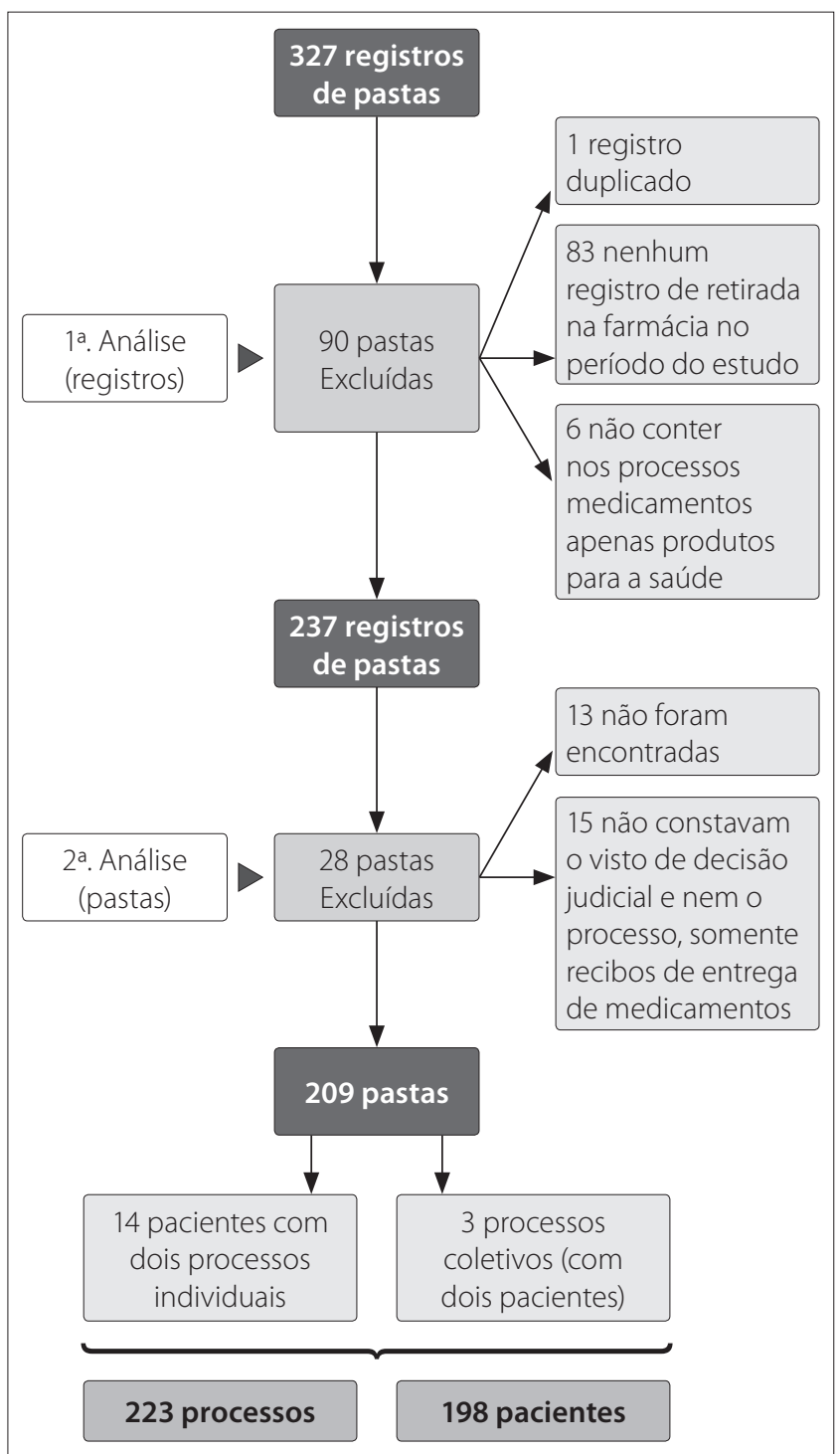

Figura 1. Fluxograma da análise dos registros e das pastas dos processos vigentes de solicitações de medicamentos em 2010, impetrados contra o Município de Tubarão - SC.

uma classificação nossa por se tratar de outras situações não classificadas, como por exemplo, doenças diversas, idade avançada e velhice. Entre os problemas de saúde, os mais comuns foram: doenças do aparelho circulatório (26,7\%); transtornos mentais e comportamentais $(26,4 \%)$; doenças dos olhos e anexos (8,6\%), doenças do sistema osteomuscular e do tecido conjuntivo $(8,6 \%)$ e doenças do sistema nervoso $(8,1 \%)$. As neoplasias foram descritas em $1,8 \%$ dos processos judiciais analisados.

A Tabela 2 apresenta o perfil dos medicamentos solicitados quanto ao registro na Anvisa e a presença em listas padronizadas. Observou-se que, dos medicamentos solicitados, $42,5 \%$ pertenciam às listas padronizadas de medicamentos fornecidos pelo Município, pelo Estado ou pela União. 
Tabela 1. Características dos processos judiciais de solicitações de medicamentos impetrados contra o Município de Tubarão - SC, em 2010. n=223.

\begin{tabular}{|c|c|c|}
\hline Variáveis & Descrição & n (\%) \\
\hline \multirow[t]{4}{*}{ Réu das ações } & Município & $170(76,2)$ \\
\hline & Município e Estado & $46(20,6)$ \\
\hline & $\begin{array}{l}\text { Município, } \\
\text { Estado e União }\end{array}$ & $1(0,5)$ \\
\hline & Indeterminado' & $6(2,7)$ \\
\hline \multirow{3}{*}{$\begin{array}{l}\text { Representação jurídica } \\
n=203\end{array}$} & Pública & $3(1,3)$ \\
\hline & Privada & $200(89,7)$ \\
\hline & Indeterminado 2 & $20(9,0)$ \\
\hline \multirow[t]{3}{*}{ Vínculo do prescritor } & Público & $68(30,5)$ \\
\hline & Privado & $123(55,2)$ \\
\hline & Indeterminado 3 & $32(14,3)$ \\
\hline \multirow{2}{*}{$\begin{array}{l}\text { Tempo de vigência } \\
\text { do processo }\end{array}$} & Determinado & $0(0,0)$ \\
\hline & Indeterminado & $223(100,0)$ \\
\hline \multirow{2}{*}{$\begin{array}{l}\text { Quantidade de processos } \\
\text { com tutela antecipada }\end{array}$} & Sim & $198(88,8)$ \\
\hline & Indeterminado 4 & $25(11,2)$ \\
\hline \multirow{2}{*}{$\begin{array}{l}\text { Processos com descrição } \\
\text { do problema de saúde }\end{array}$} & Sim & $221(99,1)$ \\
\hline & Não & $2(0,9)$ \\
\hline \multirow{2}{*}{$\begin{array}{l}\text { Processos com } \\
\text { descrição da CID } 10\end{array}$} & Sim & $159(71,3)$ \\
\hline & Não & $64(28,7)$ \\
\hline
\end{tabular}

1 Pastas com processos sem a folha da petição inicial.

2 Não constavam a petição do representante legal do paciente e nem a procuração que pudesse identificar o procurador da parte.

3 Apresentavam cópia das prescrições com a identificação do Serviço de Saúde ilegível ou sem esta informação.

4 Pastas desses processos sem o visto de decisão judicial completo

Nos processos analisados, foram solicitados 773 medicamentos, para 198 pacientes, o que confere uma média por paciente de 3,9 (DP: $\pm 2,7)$ medicamentos. Destes medicamentos, 90,1\% estão distribuídos em seis classes terapêuticas segundo a classificação ATC, apresentadas na Tabela 3.

Segundo dados fornecidos pela Controladoria Geral do Município, foram gastos com a aquisição de medicamentos do Componente Básico da Assistência farmacêutica em 2010 cerca de $\mathrm{R} \$ 9,00$ por habitante/ano. Já para atender as demandas judiciais em 2010, a média de gasto por paciente correspondeu a $\mathrm{R} \$ 1.846,24$, ou seja, aproximadamente 200 vezes mais do que o primeiro. O que corresponde a um gasto total no ano de 2010 de $\mathrm{R} \$ 365.555,52$ por esta via de acesso no município.

Na Tabela 4 estão apresentados os medicamentos solicitados categorizados segundo o grupo anatômico no qual atuam (classificação ATC). Para cada grupo de medicamentos foram realizadas diversas análises, as quais se encontram apresentadas em porcentagem. Neste contexto foi estimado o percentual de pacientes atendidos, do gasto registrado para a aquisição dos medicamentos por parte do município,
Tabela 2. Medicamentos solicitados via judicial contra o município no ano de 2010 quanto ao registro na ANVISA, data de registro, prescrição pelo nome genérico e a presença em listas padronizadas de medicamentos, $n=773$

\begin{tabular}{|c|c|c|}
\hline Variáveis & Descrição & $n(\%)$ \\
\hline \multicolumn{3}{|l|}{ Registro na Anvisa } \\
\hline \multirow[t]{2}{*}{ Presença de Registro } & Medicamentos registrados & $766(99,1)$ \\
\hline & $\begin{array}{l}\text { Medicamentos não } \\
\text { encontrados }\end{array}$ & $7(0,9)$ \\
\hline \multirow[t]{2}{*}{ Data de Registro } & Medicamentos inovadores* & $71(9,2)$ \\
\hline & $\begin{array}{l}\text { Medicamentos (superior } \\
\text { a cinco anos) }\end{array}$ & $702(90,8)$ \\
\hline \multicolumn{3}{|c|}{ Prescrição por nome genérico } \\
\hline \multicolumn{2}{|l|}{ Sim } & $243(31,4)$ \\
\hline \multicolumn{2}{|l|}{ Não } & $530(68,6)$ \\
\hline \multicolumn{3}{|c|}{ Presença dos Medicamentos em listas padronizadas } \\
\hline \multirow[t]{2}{*}{$\begin{array}{l}\text { Programa Farmácia } \\
\text { Popular }\end{array}$} & $\begin{array}{l}\text { Presentes ou parcialmente } \\
\text { presentes** }^{* *}\end{array}$ & $32(4,2)$ \\
\hline & Não Presentes & $741(95,8)$ \\
\hline \multirow[t]{2}{*}{ CEAF } & $\begin{array}{l}\text { Presentes ou parcialmente } \\
\text { presentes }\end{array}$ & $78(10,1)$ \\
\hline & Não Presentes & $695(89,9)$ \\
\hline \multirow[t]{2}{*}{ RENAME } & $\begin{array}{l}\text { Presentes ou parcialmente } \\
\text { presentes }\end{array}$ & $332(43,0)$ \\
\hline & Não Presentes & $441(57,0)$ \\
\hline \multirow[t]{2}{*}{ REMUME } & $\begin{array}{l}\text { Presentes ou parcialmente } \\
\text { presentes }\end{array}$ & $250(32,4)$ \\
\hline & Não Presentes & $523(67,6)$ \\
\hline
\end{tabular}

*Registro do medicamento com data inferior a cinco anos; ** Parcialmente presentes - são medicamentos cujo princípio ativo está disponível, mas não confere a concentração ou forma farmacêutica.

da quantidade disponibilizada nas várias listas padronizadas, bem como, dos medicamentos considerados novos.

Avaliando os dez medicamentos que representaram maior gasto, observou-se a importância da contribuição dos medicamentos antineoplásicos e imunomoduladores (50\% do gasto total). Também se verificou que dez especialidades (de um total de 392 ) representam $61,5 \%$ do gasto com medicamentos judiciais no ano de estudo. Além disso, destaca-se que $32,6 \%$ desses gastos são com medicamentos constantes na lista do CEAF.

\section{Discussão}

Inicialmente é importante salientar que conhecer o perfil dos medicamentos solicitados via judicial deve levar a discussão de quais medidas podem ser adotadas para garantir o direito ao acesso e melhorar a gestão da assistência farmacêutica. Neste sentido é importante destacar que o financiamento do Componente Básico da Assistência Farmacêutica (CBAF) é de responsabilidade das três esferas de governo, sendo apenas 25\% de responsabilidade do município. As ações judiciais 
Tabela 3. Medicamentos solicitados nos processos judiciais, vigentes em 2010, impetrados contra o Município de Tubarão - SC classificados segundo a classificação.

\begin{tabular}{|c|c|c|c|}
\hline Grupo anatômico Principal* & $\mathrm{n}(\%)$ & Principais representantes & n (\%) \\
\hline \multirow[t]{4}{*}{ A - Aparelho digestivo e metabolismo } & \multirow[t]{4}{*}{$68(8,8)$} & Carbonato de Cálcio & $14(20,6)$ \\
\hline & & Omeprazol & $12(17,6)$ \\
\hline & & Insulinas & $10(14,7)$ \\
\hline & & Outros & $32(47,1)$ \\
\hline \multirow[t]{4}{*}{ B - Sangue e órgão hematopoiéticos } & \multirow[t]{4}{*}{$52(6,7)$} & Ácido Acetilsalicílico & $19(36,5)$ \\
\hline & & Clopidrogrel & $16(30,8)$ \\
\hline & & Cilostazol & $9(17,3)$ \\
\hline & & Outros & $8(15,4)$ \\
\hline \multirow[t]{5}{*}{ C - Aparelho Cardiovascular } & \multirow[t]{5}{*}{$224(28,9)$} & Metoprolol & $21(9,4)$ \\
\hline & & Propatilnitrato & $16(7,1)$ \\
\hline & & Losartana & $16(7,1)$ \\
\hline & & Sinvastatina & $16(7,1)$ \\
\hline & & Outros & $155(69,3)$ \\
\hline \multirow[t]{3}{*}{ D - Medicamentos dermatológicos } & \multirow[t]{3}{*}{$4(0,5)$} & Acitretina & $2(50,0)$ \\
\hline & & Butenafina & $1(25,0)$ \\
\hline & & Desonida & $1(25,0)$ \\
\hline \multirow[t]{3}{*}{ G - Aparelho genito-urinário e hormonas sexuais } & \multirow[t]{3}{*}{$8(1,1)$} & Estradiol & $3(37,5)$ \\
\hline & & Finasterida & $3(37,5)$ \\
\hline & & Outros & $2(25,0)$ \\
\hline \multirow{3}{*}{$\begin{array}{l}\text { H - Preparados hormonais sistémicos, } \\
\text { excluindo hormonas sexuais }\end{array}$} & \multirow[t]{3}{*}{$13(1,7)$} & Levotiroxina Sódica & $9(69,2)$ \\
\hline & & Deflazacort & $3(23,1)$ \\
\hline & & Outros & $1(7,7)$ \\
\hline \multirow[t]{2}{*}{ J - Anti-infecciosos gerais para uso sistêmico } & \multirow[t]{2}{*}{$2(0,3)$} & Fluconazol & $1(50,0)$ \\
\hline & & Vacina Anti HPV (quadrivalente) & $1(50,0)$ \\
\hline \multirow[t]{4}{*}{ L - Agentes antineoplásicos e imunomoduladores } & \multirow[t]{4}{*}{$18(2,3)$} & Infliximab & $5(27,8)$ \\
\hline & & Metotrexato & $3(16,7)$ \\
\hline & & Azatioprina & $3(16,7)$ \\
\hline & & Outros & $7(38,9)$ \\
\hline \multirow[t]{4}{*}{ M - Sistema musculo-esquelético } & \multirow[t]{4}{*}{$35(4,5)$} & Ciclobenzaprina & $5(14,3)$ \\
\hline & & Meloxican & $3(8,6)$ \\
\hline & & Alopurinol & $3(8,6)$ \\
\hline & & Outros & $24(68,5)$ \\
\hline \multirow[t]{4}{*}{$\mathrm{N}$ - Sistema Nervoso } & \multirow[t]{4}{*}{$287(37,1)$} & Clonazepam & $33(11,5)$ \\
\hline & & Sertralina & $19(6,6)$ \\
\hline & & Ácido Valpróico & $18(6,3)$ \\
\hline & & Outros & $217(75,6)$ \\
\hline P - Produtos antiparasitários, insecticidas e repelentes & $3(0,4)$ & Hidroxicloroquina & $3(100,0)$ \\
\hline \multirow[t]{3}{*}{ R - Aparelho Respiratório } & \multirow[t]{3}{*}{$21(2,7)$} & Formeterol e budesonida & $5(23,8)$ \\
\hline & & Brometo de tiotrópio & $5(23,8)$ \\
\hline & & Outros & $11(52,4)$ \\
\hline \multirow[t]{3}{*}{ S - Órgãos dos sentidos } & $28(3,6)$ & Dorzolomida & $5(17,9)$ \\
\hline & & Latanoprost & $5(17,9)$ \\
\hline & & Outros & $18(64,2)$ \\
\hline V-Vários & $2(0,3)$ & Deferasirox & $2(100,0)$ \\
\hline Não Classificados & $8(1,1)$ & Isoflavona & $1(12,5)$ \\
\hline & & Melilotus officinalis & $1(12,5)$ \\
\hline & & Gotas Umidificantes & $6(75,0)$ \\
\hline TOTAL & $773(100,0)$ & & \\
\hline
\end{tabular}

*neste caso foi adotada a classificação ATC, o que nem sempre está de acordo com a indicação terapêutica adotada pelo paciente. 
Tabela 4. Categorização dos medicamentos, solicitados nos processos contra o município em 2010, pelo grupo anatômico principal da Classificação ATC e sua relação (expressa em porcentagem) com o gasto registrado, presença em listas padronizadas, classificação como medicamento novo e número de pacientes atendidos.

\begin{tabular}{|c|c|c|c|c|c|c|c|}
\hline \multirow{2}{*}{$\begin{array}{l}\text { Classificação } \\
\text { ATC }\end{array}$} & \multirow{2}{*}{$\begin{array}{c}\text { Gasto } \\
\text { Registrado (\%) }\end{array}$} & \multicolumn{4}{|c|}{ Presença em listas padronizadas } & \multirow{2}{*}{$\begin{array}{l}\text { Medicamentos } \\
\text { novos (\%) }\end{array}$} & \multirow{2}{*}{$\begin{array}{c}\text { Pacientes } \\
\text { atendidos } *(\%)\end{array}$} \\
\hline & & REMUME (\%) & CEAF (\%) & RENAME (\%) & F. Popular (\%) & & \\
\hline A & 6,6 & 0,4 & 0,0 & 1,0 & 0,1 & 0,9 & 22,2 \\
\hline B & 1,5 & 1,0 & 0,0 & 0,3 & 0,0 & 0,0 & 19,8 \\
\hline C & 10,5 & 1,2 & 1,9 & 3,3 & 0,1 & 3,0 & 40,1 \\
\hline D & 0,2 & 0,0 & 0,1 & 0,0 & 0,0 & 0,0 & 1,9 \\
\hline G & 0,5 & 0,0 & 0,0 & 0,0 & 0,0 & 0,0 & 3,8 \\
\hline $\mathrm{H}$ & 0,4 & 0,1 & 0,0 & 0,1 & 0,0 & 0,1 & 5,7 \\
\hline J & 0,0 & 0,0 & 0,0 & 0,0 & 0,0 & 0,0 & 0,9 \\
\hline L & 50,0 & 0,0 & 25,0 & 0,7 & 0,0 & 4,4 & 8,0 \\
\hline$M$ & 3,1 & 0,2 & 0,0 & 0,2 & 0,0 & 0,3 & 12,7 \\
\hline $\mathbf{N}$ & 17,4 & 2,9 & 5,1 & 3,9 & 0,0 & 2,1 & 57,5 \\
\hline $\mathbf{P}$ & 0,1 & 0,0 & 0,1 & 0,1 & 0,0 & 0,0 & 1,4 \\
\hline $\mathbf{R}$ & 2,2 & 0,3 & 0,4 & 0,2 & 0,0 & 0,0 & 6,1 \\
\hline$S$ & 3,0 & 0,0 & 0,0 & 0,0 & 0,0 & 0,8 & 10,4 \\
\hline V & 4,2 & 0,0 & 4,2 & 0,0 & 0,0 & 4,2 & 0,5 \\
\hline NC & 0,2 & 0,0 & 0,0 & 0,0 & 0,0 & 0,1 & 1,9 \\
\hline Total & 100,0 & 5,9 & 36,8 & 9,8 & 0,2 & 16,0 & - \\
\hline
\end{tabular}

*a somatória desta coluna não totaliza 100\% pelo fato de um mesmo paciente poder solicitar mais de um medicamento por processo judicial. $C E A F=$ Componente


Medicamentos dermatológicos; $\mathrm{G}=$ Aparelho geniturinário e hormônios sexuais; $\mathrm{H}=$ Preparados hormonais sistêmicos e, excluindo hormônios sexuais; $\mathrm{J}=$ Antiinfecciosos gerais para uso sistêmico; $\mathrm{L}=$ Agentes antineoplásicos e imunomoduladores; $\mathrm{M}=$ Sistema musculoesquelético; $\mathrm{N}=$ Sistema Nervoso; $\mathrm{P}=$ Produtos antiparasitários, inseticidas e repelentes; $\mathrm{R}=$ Aparelho respiratório; $S=$ Órgãos dos sentidos; $V=$ Vários; $N C=$ Não classificados.

aqui avaliadas foram demandas contra o município, o que representa um custo ainda maior para o gestor municipal. Além disso, o gasto com o CBAF está previsto no orçamento do município, o que já não ocorre com os medicamentos solicitados sob a via judicial. Isto é ainda mais pertinente quando se observa que pouco mais de 200 pacientes representaram um gasto de aproximadamente $50 \%$ do valor gasto com toda a população do município (98.000) no ano de estudo considerando os recursos empenhados no CBAF.

Muitas são as situações que contribuem para o maior custo dos medicamentos por esta via, entre elas pode-se citar que: os medicamentos inovadores possuem um único laboratório produtor e o monopólio sobre o preço; as compras não são programadas e por isso os preços de compras podem ser ainda mais elevados; como os medicamentos não são padronizados a quantidade comprada é muito pequena o que também pode elevar os preços dos medicamentos. Além disso, deve-se salientar que, como muitas vezes este paciente não é acompanhado pela equipe multidisciplinar, não há registros da adesão ao tratamento nem das possíveis modificações do tratamento. Ou seja, é possível que o município compre e o paciente pare de utilizar.

A judicialização do acesso aos medicamentos parece ser catastrófica em termos de orçamento para os municípios.
Pior ainda quando essa via á utilizada para garantir o acesso daqueles medicamentos que deveriam ser fornecidos e não estão disponíveis. Por não estarem previstos no planejamento da assistência farmacêutica municipal, os gastos são cada vez maiores com medicamentos adquiridos em consequência de uma ação judicial podem gerar descontrole orçamentário.

O perfil dos processos identificados no presente estudo assemelha-se a outras realidades já descritas no Brasil (Pereira et al, 2010; Ronsein, 2010; Vieira \& Zucchi, 2009), onde a representação jurídica predominante é a particular. Da mesma forma, os prescritores são em sua maioria vinculados ao serviço privado. Esse dado é importante, visto que a prescrição médica é praticamente a única base para o deferimento das ações judiciais (Galato et al, 2012). Neste sentido, é necessária a convergência entre a prática clínica e a gestão de saúde pública, uma vez que há um grande número de medicamentos disponibilizados no sistema público que poderiam ser utilizados com igual eficácia e segurança e a busca desta informação no momento da prescrição de um medicamento pode evitar a demanda judicial (Pepe et al, 2010; Galato et al, 2012; Figueiredo, Pepe, Osorio-de-Castro, 2010). Além disso, usuários que possuem mais acesso à informação ou recursos financeiros para pagar advogados são privilegiados no fornecimento de 
medicamentos via ação judicial em detrimento de segmentos de usuários que têm mais necessidades (Vieira, 2008).

Um dos critérios na busca da racionalidade nos processos é descrito por Galato et al. (2012), que seria a criação de comissões de peritos para reavaliação dos pacientes em períodos regulares durante o fornecimento dos medicamentos pela via judicial, visto que, dos processos analisados, nenhum apresentava prazo determinado para o fornecimento dos medicamentos.

Vieira e Zuchi, que realizaram seu trabalho com os processos impetrados contra a Secretaria de Saúde da cidade de São Paulo, identificaram que a maioria das ações foi deferida com antecipação de tutela, como também observada na presente pesquisa, ressaltando que, mesmo com direito a defesa, o que importa é que o fato já está consumado com a tutela antecipada, e, portanto, mesmo que se chegue à conclusão de que a demanda não se justificava, os recursos públicos já foram despendidos.

Apenas um terço dos medicamentos foi prescrito pelo nome genérico, como determina a Lei Federal no 9787/1999. A adoção da denominação genérica, ou seja, da prescrição pela denominação comum brasileira ou denominação comum internacional, para as prescrições de medicamentos no SUS é obrigatória. Portanto, uma vez que os mandatos judiciais foram impetrados contra o serviço público municipal de saúde, esta obrigatoriedade deveria ter sido observada. A prescrição por nome genérico, entre outras vantagens, pode possibilitar a redução dos gastos na aquisição dos medicamentos.

Além desta limitação na nomenclatura para solicitar os medicamentos, foram observadas deficiências na sua composição, como ausência dos dados do paciente ou da situação clínica, que dificultam a avaliação da racionalidade da medicação prescrita. Além disso, os processos não trazem o histórico farmacoterapêutico ou clínico do paciente, o que, por vezes, poderia justificar a adoção da prescrição que deu origem ao processo, conforme destacado por Galato et al. (2012).

Somado a isso, a elevada frequência de medicamentos pertencentes a listas padronizadas, realidade também identificada em outras pesquisas (Macedo, Lopes \& Barberato-FiIho, 2011; Messeder, Osorio-de-Castro \& Luiza, 2005; Romero, 2008), pode ser resultado da falta de comunicação entre o setor público e o privado de saúde, bem como, representação de um problema de gestão da assistência farmacêutica decorrente da falha de fornecimento desses medicamentos (Pandolfo, Delduque \& Amaral, 2012; Reveiz et al, 2013). Contudo, há de se cogitar que existe a possibilidade de razões culturais para esta realidade. Estas razões podem estar relacionadas à comodidade da retirada dos medicamentos em um único setor (visto que os processos são geralmente compostos por um elenco de medicamentos padronizados e não padronizados e que podem ser distribuídos em diferentes locais) e também existe a possibilidade de que alguns pacientes sintam-se expostos e desconfortáveis em exercer o direito de acesso aos medicamentos padronizados nas unidades de saúde.

Em relação aos gastos, observou-se que entre os grupos anatômicos da ATC, os medicamentos que representam maior percentual de gasto com a via de acesso judicial foram os medicamentos antitumorais e imunomoduladores. Estes medicamentos também foram destacados em outros estudos como as classes mais onerosas (Ronsein, 2010) e há inclusive evidências em alguns locais de influência da indústria de medicamentos na solicitação destes medicamentos por via judicial (Campos-Neto et al, 2012). Por outro lado, os antitumorais não estão incluídos em nenhuma lista de medicamentos, seu acesso ocorre por meio da autorização de procedimentos de alto custo e por procedimento.

Um ponto interessante que merece destaque é o fato de que os medicamentos do sistema nervoso são os mais demandados, não representam os medicamentos novos e não possuem alto custo, mas possuem baixa cobertura nas listas. Este fato deve ser analisado com maior cautela.

Em relação à essencialidade, avaliada neste contexto pela participação nas listas da RENAME e REMUME, observamos que um valor menor que $10 \%$ está presente na lista nacional e, neste caso, menos de $6 \%$ dos gastos poderiam ter sido reduzidos se os autores das ações tivessem utilizado a farmácia básica do município para acessar os medicamentos solicitados. Contudo, verificou-se que medicamentos pertencentes ao CEAF oneram em quase $40 \%$ os gastos pela via judicial. Neste caso, existem pelo menos três situações possíveis, pacientes e prescritores não conhecem a via de acesso aos medicamentos pelo CEAF, as exigências do CEAF tais como a prescrição por um especialista e exames de monitoramento são difíceis de cumprir e a indicação clínica para o autor da ação não está prevista no protocolo do CEAF. Para responder melhor a estas questões, seria necessária uma revisão desses processos.

Outro dado interessante é a participação dos medicamentos novos que representam 16\% das solicitações, o que pode refletir na influência da indústria. Recentemente, um trabalho desenvolvido por Campos-Neto et al. (2012) demonstrou indícios de que a indústria de medicamentos pode influenciar os médicos e, consequentemente, a justiça no acesso aos medicamentos por meio de ações judiciais. Neste contexto, as informações constantes nos processos quanto ao perfil dos pacientes, elementos processuais, problemas de saúde e medicamentos demonstram possível uso irracional de medicamentos, o que pode estar relacionado ao gasto tão superior por esta via de acesso. Além disso, os resultados encontrados apontam para a necessidade de se inserir a avaliação econômica para a tomada de decisão dos gestores da saúde, auxiliando o município, Comissão de Farmácia e Terapêutica e os prescritores a conciliarem as necessidades terapêuticas com as possibilidades de custeio, oferecendo ao 
paciente o melhor tratamento com o menor custo possível, em prol da coletividade.

Contudo, deve-se pontuar que esta via de acesso é adequada para garantir acesso aos medicamentos em diferentes situações. No entanto, deve adotar critérios que não garantam apenas o acesso aos medicamentos, mas sim o seu acesso e uso racional (Prado, 2013).

Uma limitação desta pesquisa está relacionada ao tipo de estudo adotado, que se caracteriza por uma análise retrospectiva baseada em documentos, o que levou a perda de alguns processos durante a análise. Outra limitação refere-se ao fato de não ter sido avaliado se os pacientes que impetraram as ações analisadas poderiam ser contemplados pelos PCDT, e, portanto, ter acesso aos medicamentos presentes na lista do Componente Especializado da Assistência Farmacêutica. No entanto, os achados aqui apresentados trazem contribuições importantes para a reflexão deste fenômeno observado no Brasil.

\section{Conclusões}

A solicitação de medicamentos via judicial tem um custo médio por paciente muito mais elevado que os medicamentos do Componente Básico da Assistência Farmacêutica e parte deste custo pode estar associado à solicitação de medicamentos novos, bem como, com antineoplásicos e imunossupressores.

A presença de muitos medicamentos que participam de listas padronizadas gera a discussão sobre a garantia de acesso pelo sistema público, bem como gera a hipótese de que a facilidade de acesso em um único local possibilite a construção de processos com medicamentos padronizados e não padronizados.

Corroborando com este cenário, a falta de informações nos processos, em especial da situação de saúde atual do paciente e da história farmacoterapêutica, impede que uma avaliação mais adequada da racionalidade das solicitações de medicamentos realizadas.

\section{Referências bibliográficas}

Biehl J, Amons JJ, Socal MP, Petryna A. Between the court and the clinic: lawsuits for medicines and the right to health in Brazil. Health Hum. Rights. 2012;14(1):E36-52.

Campos-Neto OH, Acurcio FA, Machado MAA, Ferré F, Barbosa FLV, Cherchiglia ML, Andrade ELG. Doctors, lawyers and pharmaceutical industry on health lawsuits in Minas Gerais, Southeastern Brazil. Rev. Saude Publica. 2012;46(5):784-90.

Figueiredo TA, Pepe VLE, Osorio-de-Castro CGS. Um enfoque sanitário sobre a demanda judicial de medicamentos. Physis. 2010;20:101-18.

Galato D, Nesi H, Rosso JA, Oliveira CRSM, Trauthman SC, Lunardi T, SchuelterTrevisol F. Judicialização de medicamentos no Brasil: um processo que deve ser racionalizado. Rev. Saude Publica. 2012;46:390-1.
Leite SN, Pereira SMP, Silva P, Nascimento-Jr JM, Cordeiro BC, Veber AP. Ações Judiciais e demandas administrativas na garantia do direito de acesso a medicamentos em Florianópolis-SC. Rev. Direito Sanit. 2009;10(2):13-28.

Macedo EL, Lopes LC, Barberato-Filho S. Análise técnica para a tomada de decisão do fornecimento de medicamentos pela via judicial. Rev. Saude Publica. 2011;45:706-13.

Messeder AM, Osorio-de-Castro CGS, Luiza VL. Mandados judiciais como ferramenta para garantia do acesso a medicamentos no setor público: a experiência do Estado do Rio de Janeiro, Brasil. Cad. Saude Publica. 2005;21:525-34.

Ministério da Saúde. Lei no 9.787, de 10 de fevereiro de 1999. Altera a Lei Altera nº.360, de 23 de setembro de 1976, que dispõe sobre a vigilância sanitária estabelece o medicamento genérico, dispõe sobre a utilização de nomes genéricos em produtos farmacêuticos e dá outras providências. [Acessado 2015 ago 09]. Disponível em: http://www. planalto.gov.br/ccivil_03/leis/19787.htm.

Paim J, Travassos C, Almeida C, Bahia L, Macinko J. The Brazilian health system: history, advances, and challenges. Lancet. 2011; 21;377(9779):1778-97.

Pandolfo M, Delduque MC, Amaral RG. Aspectos jurídicos e sanitários condicionantes para o uso da via judicial no acesso aos medicamentos no Brasil. Rev. Salud Publica. 2012;14(2):340-9.

Pepe VLE, Figueiredo TA, Simas L, Osorio-de-Castro CG, Ventura M. A judicialização da saúde e os novos desafios da gestão da assistência farmacêutica. Cien. Saude Colet. 2010; 15(5):2405-14.

Pereira JR, Santos RI, Nascimento-Junior JM, Schenkel EP. Análise das demandas judiciais para o fornecimento de medicamentos pela Secretaria de Estado da Saúde de Santa Catarina nos anos de 2003 e 2004. Cien. Saude Colet. 2010;15: 3551-60.

Prado MM. The debatable role of courts in Brazil's health care system: does litigation harm or help? J. Law Med. Ethics 2013;41(1):124-37

Reveiz L, Chapman E, Torres R, Fitzgerald JF, Mendonza A, Bolis M, Salgado O. Litigios por derecho a la salud en tres países de América Latina: revisión sistemática de la literatura. Rev. Panam. Salud Publica. 2013:33:213-22.

Romero LC. Judicialização das políticas de assistência farmacêutica: o caso do Distrito Federal. Brasília, DF: Consultoria Legislativa do Senado Federal, 2008. [Acessado 2015 ago 09]. Disponível em: http://www2. senado.leg.br/bdsf/item/id/96829

Ronsein JG. Análise do perfil das solicitações de medicamentos por demanda judicial do Estado de Santa Catarina no período de 2005 a 2008. 2010. [Dissertação]. Florianópolis (SC): Universidade Federal de Santa Catarina; 2010.

Sant'ana JMB, Pepe VLE, Figueiredo TA, Osorio-de-Castro CGS, Ventura M. Racionalidade terapêutica: elementos médico-sanitários nas demandas judiciais de medicamentos. Rev. Saude Publica. 2011;45:714-21.

Vieira FS. Ações judiciais e direito à saúde: reflexão sobre a observância aos princípios do SUS. Rev. Saude Publica. 2008;42:365-9.

Vieira FS. Gasto do Ministério da Saúde com medicamentos: tendência dos programas de 2002 a 2007. Rev. Saude Publica. 2009;43:674-81.

Vieira FS, Zucchi P. Demandas judiciais e assistência terapêutica no sistema único de saúde. Rev. Assoc. Medica Bras. 2009;55:672-83.

Vieira MRS, Hessing A, Silva DCA, Chaaban SVH, Pereira LAA. Ações judiciais como ferramenta para garantia do acesso ao medicamento: experiência de Santos - SP. J Bras Econ Saude. 2013;5(1):25-28.

World Health Organization. Anatomical Therapeutic Chemical Classification (ATC). [Acessado 2015 mai 03]. Disponível em: http://www.whocc.no/ atc/structure_and_principles. 